OPEN ACCESS

Edited by:

Juliet Willetts,

University of Technology Sydney,

Australia

Reviewed by:

Chrysoula Sfynia,

Cranfield University, United Kingdom

Cynthia Mitchell,

University of Technology Sydney,

Australia

${ }^{*}$ Correspondence:

Pippa Scott

pippa.scott@i-san.co.uk

Specialty section:

This article was submitted to Water and Wastewater Management,

a section of the journal

Frontiers in Environmental Science

Received: 13 August 2019

Accepted: 12 May 2020

Published: 03 July 2020

Citation:

Scott $P$ and Cotton AP (2020) The

Sanitation Cityscape - Toward

a Conceptual Framework

for Integrated and Citywide Urban

Sanitation. Front. Environ. Sci. 8:70.

doi: $10.3389 /$ fenvs.2020.00070

\section{The Sanitation Cityscape - Toward a Conceptual Framework for Integrated and Citywide Urban Sanitation}

\author{
Pippa Scott ${ }^{1 *}$ and Andrew P. Cotton ${ }^{2}$ \\ ${ }^{1} i$-san Partner and Principal Consultant, Edinburgh, United Kingdom, ${ }^{2}$ Independent Consultant, Shepshed, United Kingdom
}

In the last decade, the sanitation service chain model has become the de facto framework for much research and development in urban non-networked sanitation. People, their priorities and urban ways of living, as well as the conditions that underpin sustainable services, are too often overlooked in current conceptualizations of urban sanitation service delivery. This paper suggests that, as the sector moves toward a new paradigm of Citywide Inclusive Urban Sanitation, it is timely to revisit the conceptual framing of urban sanitation. The Sanitation Cityscape is a conceptual framework for citywide urban sanitation. It identifies the key factors of urban sanitation and locates those within a framework using three conceptual environments: The Living Environment, the Service Delivery Environment, and the Enabling Environment. Using a proposed set of 16 indicators and locating existing tools (for example, the Living Conditions Diamond, the fecal flow diagram, and the Citywide Service Delivery Assessment), the framework looks beyond the linear framing of sanitation services to gain a better understanding of the surrounding context and externalities. For the researcher and practitioner alike, we suggest that the Sanitation Cityscape can provide a coherent "frame" to locate the components of the urban sanitation puzzle predictably and systematically. It lends itself to rapid diagnostic analysis and more appropriate targeting of appropriate sanitation interventions. The paper includes insights from application of the Sanitation Cityscape framework including moving toward an outcome-based sanitation service delivery model, efficiencies in data collection, creating area typologies to align sanitation responses, setting enabling environment analyses boundaries purposefully and intentionally, and identifying key interfaces as potential intervention points or system levers. We hope that the Sanitation Cityscape might provide a foundation for greater consistency and a common vocabulary around the fundamental concepts and indicators relevant to urban sanitation.

Keywords: sanitation, urban, conceptual framework, urban governance, sanitation cityscape 


\section{INTRODUCTION}

\section{Overview and Problem Statement}

In the last decade, the sanitation service chain model, which articulates the typical components of fecal sludge management (FSM) i.e., the capture, storage, transport, treatment, and reuse/disposal of fecal waste, has become the de facto framework for much research and development in urban non-networked urban sanitation. This linear framing has been hugely instrumental in the past decade's advances; its simplicity and widespread adoption has catalyzed sector specialization and a granular understanding of urban sanitation. It has significantly raised the profile of non-sewered sanitation activities. Nevertheless, the focus on fecal flow mapping is insufficient to grasp the inherent complexity of human technology - environment urban sanitation systems. Previous conceptualizations in sanitation discourse, for example, Sanitation Safety Planning (WHO, 2015), CLUES (Lüthi et al., 2011), Sanitation21 (IWA, 2006; Parkinson et al., 2014), Household Centered Environmental Sanitation (Kalbermatten et al., 1999), and the Strategic Sanitation Approach (Wright, 1997), tend to have been pitched as planning tools, which are dense to grasp and have seen limited uptake. We suggest that the widespread uptake of the sanitation service chain has been due to its conceptual simplicity, from which tools and approaches have been developed.

A conceptual framework explains, either graphically or in narrative form, the main things to be studied, the key factors, concepts, or variables, and the presumed relationship between them (Miles and Huberman, 1994, p. 18). We suggest that, as the sector moves toward a new paradigm of Citywide Inclusive Urban Sanitation, the linear framing is limiting. Furthermore, there is little consensus on what a standard set of factors, concepts, and variables might be for urban sanitation. We suggest that a wider conceptual framing, one that embeds both networked and non-networked sanitation systems more explicitly within urban governance, is more appropriate to the complexities of urban service delivery. We therefore suggest that it is timely to revisit what a conceptual framework for urban sanitation might look like in an effort to consolidate sector efforts and hone the discourse vocabulary. One such model, termed the Sanitation Cityscape, builds upon past frameworks; its latest iteration is the focus of this paper.

\section{METHODS}

Jabareen (2009) describes eight iterative stages of building a conceptual framework ranging from review of existing knowledge, through identifying and categorizing key concepts; locating those within a framework; and validating the framework. Concepts are a generalized idea that may not be measurable therefore, through identifying criteria that reflects the concept, can provide a measurable indicator (what you are measuring) and research variable (how change will be measured). The focus of this paper describes identifying and categorizing key concepts and proposes a conceptual framework in which to locate them coherently. While this paper makes reference to how the framework was applied in practice, that experience is documented elsewhere (Scott and Henry, 2018) and is not the focus of this paper.

This paper begins to build a conceptual framework by identifying and categorizing key concepts for urban sanitation (Jabareen, 2009). These were identified and informed through review of recent and current urban sanitation discourse drawn from peer-reviewed and gray literature. It also draws upon previous peer-reviewed presented work of the authors reviewing the evolution of urban sanitation discourse (Scott et al., 2017) and earlier iterations by the authors of the Sanitation Cityscape framework (Scott et al., 2015, 2017; Scott, 2019).

\section{DISCUSSION}

\section{Current Urban Sanitation Discourse}

The global indicator selected by UN Member States for monitoring SDG target 6.2 "Safely managed sanitation" is the "Proportion of population using safely managed sanitation services including a handwashing facility with soap and water" where "safely managed" is defined as the use of an improved sanitation facility that is not shared with other households and where excreta is safely disposed in situ or excreta is transported and treated off-site (WHO/JMP, 2016). Measuring SDG 6.2 is problematic due to a lack of reliable measures; combing data from different source households and utilities and lack of data itself. The component parts of non-networked sanitation systems are commonly framed using the five components of the sanitation service chain: capture, containment, transport, treatment, and reuse/disposal ${ }^{1}$ (Tilley et al., 2008). The sanitation service chain has been a key area of focus in the sanitation sector in recent years and tools and standard protocols or what data types and sources are needed, such as the fecal sludge or shit-flow diagram (SFD)type analysis, for collecting evidence along these components of the chain (Shit Flow Diagram Initiative, 2018). The prevalence, the frequency, and the pathways of each of these components describe the different components of sanitation service delivery present. These provide an effective snapshot of the volumes of waste (both networked and non-networked sanitation side by side) and the array of services and infrastructure in place.

The enabling environment affects the potential to bring about effective change; it describes the set of interrelated and contextually specific functions that either facilitate or hinder sanitation service delivery, where universal access can only be sustained through well-functioning enabling environments (WSUP, 2018). There is growing consensus about what constitutes an enabling environment for sanitation, typically consisting of: policy and strategy, institutional arrangements, sector planning and monitoring, and budgeting and finance and capacity. Several tools have been developed to assess the enabling environment, including the SFD Manual

\footnotetext{
${ }^{1}$ This specifically focuses on contexts where non-networked urban sanitation is the norm, acknowledging that, for less densely populated areas, in situ treatment as well as networked sanitation (sewers) can both achieve safely managed sanitation in appropriate contexts.
} 
(Shit Flow Diagram Initiative, 2018), UNICEF Guidance on Strengthening the Enabling Environment for WASH (UNICEF, 2016), the Citywide Service Delivery Assessment (CSDA) (Peal et al., 2014; Ross et al., 2016) and CWIS CSDA review (Blackett and Hawkins, 2019), and the WSUP Conceptual Framework for Enabling Environments for Inclusive Citywide Sanitation (WSUP, 2018).

The importance of demand in sanitation programming was a major lesson to emerge from the International Decade of International Drinking Water Supply and Sanitation Decade (IDWSSD) 1981-1990 (Cairncross, 1992). Demand-led approaches to sanitation aim to create demand for improved sanitation by changing behaviors while strengthening the availability of supporting products and services. Advances in assessing demand are offering alternative experimental designs, such as discrete choice experiments, to the often-criticized contingent valuation method (CVM) techniques due to lack of accuracy and hypothetical bias (Tidwell et al., 2019). A number of theoretical models, frameworks, and decision-making tools have been developed around WASH behavior change (Dreibelbis et al., 2013) offering sanitation specific insights that demand is created when consumers have motivation (preference), opportunity, and ability to purchase sanitation technology that suits their needs (Jenkins and Scott, 2007; Devine, 2009).

\section{Limitations of Urban Sanitation Discourse}

Clearly, great progress has been made in developing tools and frameworks to understand the different components of the sanitation services delivery component parts. However, do these sufficiently describe the urban sanitation landscape as a whole? Does the de facto sanitation service chain framework sufficiently capture the key concepts to be studied? We suggest it does not, that a graphical representation of a linear chain means the enabling environment (i.e., the conditions that underpin sustainable services) and the people, and their inherent priorities, behaviors, interests, and ways of living, are too often missing or overlooked current conceptualizations of urban sanitation service delivery. Furthermore, when looking through the lens of service delivery at either the enabling environment or people, it is not uncommon to assume a narrow single-sector view of those issues, whereas due to their very nature, they are wider.

The reality of urban living (and not just low income) introduces complexities around the demand for, and the provision of, basic services. The services themselves are physically interlinked; sustainable planning for one service (e.g., sanitation) cannot proceed in isolation from others, e.g., water supply, drainage, and/or solid waste management (Cotton and Franceys, 1987, 1991). At the consumer side, demand for urban services manifests in a more nuanced way than isolated single-sector interventions; residents seek improvements across the totality of their lives (Kar, 1997; Scott et al., 2017). For sanitation, the (often latent) nature of the demand and the way issues such as space, tenure, and road access influence the planning and uptake (IWA, 2006; Tidwell et al., 2019) further compound the modes of implementation for sanitation compared to other infrastructure and services. The unit of measurement of urban living is complicated where boundaries of urban households are blurred; it is not uncommon across the world for several urban "household" units to live under one roof or on one plot; adjacent urban households often share infrastructure (such as toilets, taps, or solid waste bins) ${ }^{2}$. Enabling environment analysis, through a single-sector lens, fails to take into account wider urban governance issues, priorities, and challenges across the many facets of urban development.

\section{The Sanitation Cityscape Framework}

Current Urban Sanitation Discourse and Limitations of Urban Sanitation Discourse draw upon key factors, concepts, variables, and tools that are relevant to urban sanitation. We suggest that the application of these tools without understanding other parts of the urban system are, at best, capturing part of the story and, at worst, could do harm. We therefore suggest that a stronger conceptual framework for urban sanitation would aggregate a set of factors into key concepts and locate them, and the appropriate tools, with a wider analytical framework. The aggregate concepts we suggest can be articulated in three conceptual environments:

- The living environment is about people. It describes the domestic and peridomestic characteristics private sphere within which households make decisions. Demand and behaviors are key components here. The living environment includes sanitation but acknowledges that any given urban household, residents' priorities are heavily influenced by the living conditions that surround it and that demand for urban services manifests in a more nuanced way than single-sector interventions.

- The service delivery environment describes the functions of basic services and their infrastructure that deliver services to households. For sanitation inquiry, the service delivery environment is where the nature and mechanisms of service delivery can be focused upon. Importantly, in the framework, sanitation services are located alongside other basic services acknowledging the fundamental physical interlinkages of water supply, drainage, solid waste management, and other basic services and provide a potential placeholder to align and integrate with adjacent services.

- The enabling environment describes the wider structural and institutional context that frame overall service delivery such as policy and strategy, institutional arrangements, sector planning and monitoring, and budgeting and finance and capacity.

- The interfaces and the nature of relationships describes the relationships within and between each of these three conceptual environments, highlighting interfaces, gaps, and intervention opportunities.

We suggest that these three conceptual environments of the Sanitation Cityscape, drawn together, provide a useful analytical framework for urban sanitation systems (see Figure 1).

${ }^{2}$ This zone beyond the household, the area located near but not within the dwelling walls, is termed peridomestic zone (Sanitation 21, 2006). 


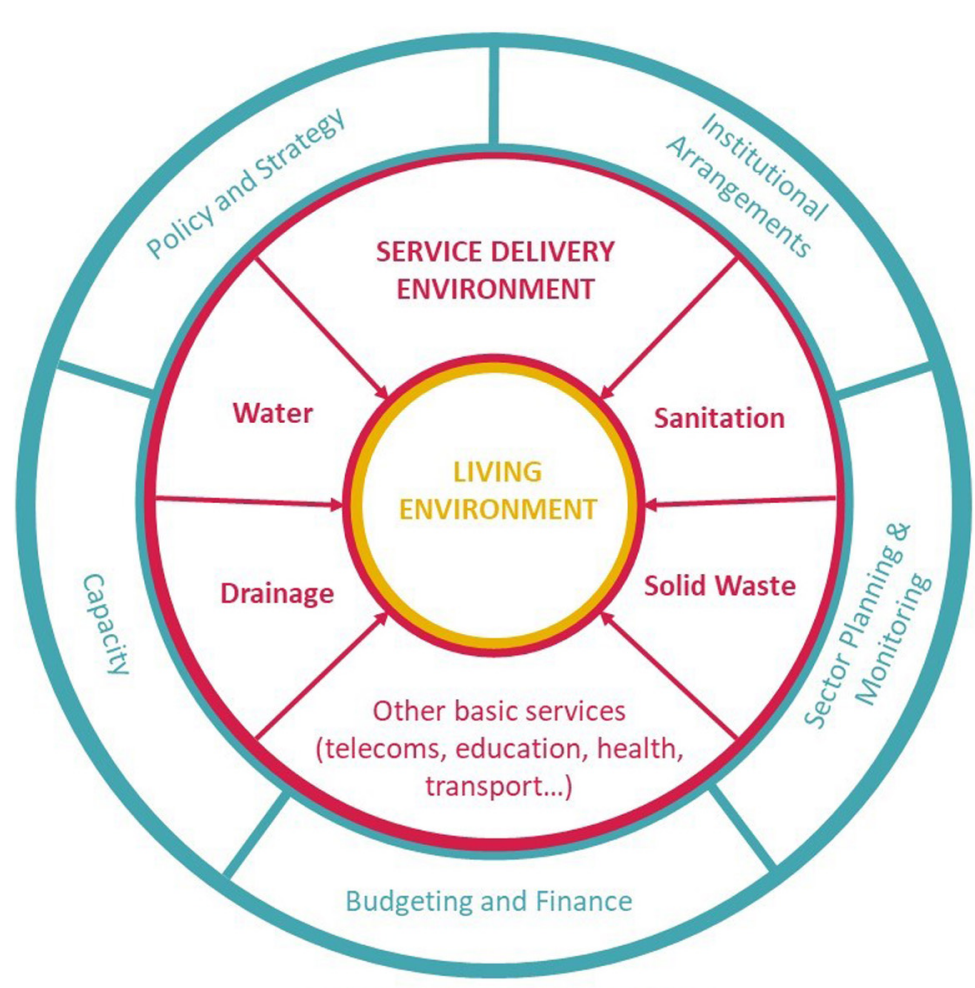

THE ENABLING ENVIRONMENT

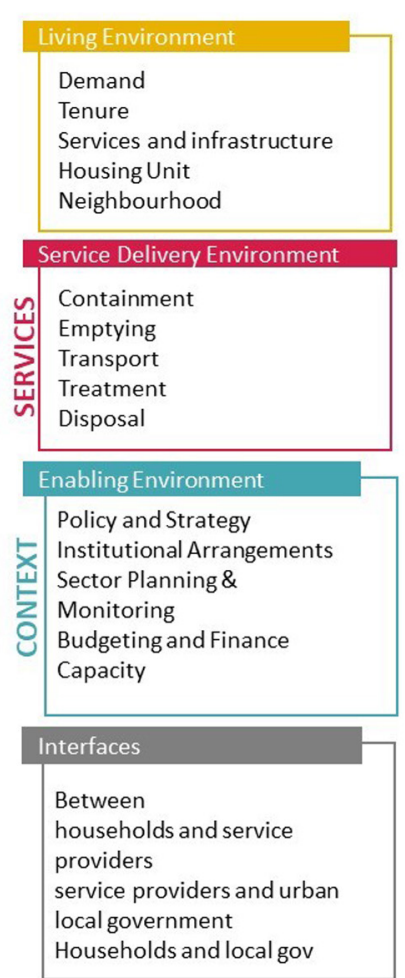

FIGURE 1 | The Sanitation Cityscape conceptual framework.

The framework can provide coherence and structure to locate granular detail within a much more complex system, allowing for specific parts of sanitation service delivery to be the focus of inquiry. In the following section, 16 core indicators are proposed to describe these three conceptual environments and the relationships between them. Figure 2 gives an overview of the main indicators we suggest could be understood in each conceptual environment and why they are relevant. It also suggests some tools and ways to measure the proposed indicators. Neither the framework itself nor the proposed indicator list are intended to be prescriptive. Different framing or applications may include other indicators, but we suggest that the approach of locating the indicators and the tools being applied within the conceptual environments is a useful one.

\section{The Living Environment}

The Living Environment describes people, their behaviors, and the peridomestic environment. Placing the Living Environment at the core of the framework echoes the development rationale of centering frameworks on the main development objective (Chambers, 1983) and earlier concentric sanitation frameworks ${ }^{3}$. We suggest that, at the Living Environment level, it is important to understand what citizens want and what their development

\footnotetext{
${ }^{3}$ Such as Sanitation 21 (IWA, 2006; Parkinson et al., 2014), Household Centered Environmental Sanitation (Kalbermatten et al., 1999), Strategic Sanitation Approach (Wright, 1997), and CLUES (Lüthi et al., 2011).
}

priorities are (for which we term an indicator demand). We note that demand often does not manifest by sector, rather people seek improvements of their overall living conditions (Kar, 1997; Scott et al., 2017); assessment of demand should not be blinkered to demand for sanitation only. To understand the living conditions of any urban settlement, the Living Conditions Diamond is a useful tool (Gulyani and Basset, 2010). It describes the living environment of any given settlement, using only four variables: tenure, housing unit, infrastructure, and neighborhood. Plotting each variable on an axis, it generates diamond profiles offering an objective comparability between settlement types both within and between urban environments. We therefore suggest that the Living Environment can be captured using five core indicators ${ }^{4}$ : the four indicators of the living conditions diamond tenure, housing unit, infrastructure, and neighborhood, as a comparable composite indicator of the living conditions of any urban settlement, with the addition of demand to reflect resident's development priorities. Measuring these five indicators, we suggest, would provide an insightful view of was happening in an urban Living Environment (see Figure 2).

\section{The Service Delivery Environment}

The five components of the sanitation service chain: capture, containment, transport, treatment, and reuse/disposal provide

\footnotetext{
${ }^{4}$ In addition to appropriate socioeconomic indicators such as age, gender, income,
} etc., as relevant. 


\begin{tabular}{|c|c|c|c|c|}
\hline \multicolumn{2}{|c|}{ WHAT? Indicators } & WHY? Why are these important? & HOW? Suggested variables & TOOLS \\
\hline \multicolumn{5}{|c|}{1 THE LIVING ENVIRONMENT } \\
\hline 1.1 & Demand & To understand residents' priorities and behaviours & $\begin{array}{l}\text { Development priorities of residents; satisfaction levels; willingness to pay; } \\
\text { specific behaviours }\end{array}$ & $\begin{array}{l}\text { Sani FOAM (Devine 2009); Discrete } \\
\text { Choice Experiments (Tidwell et al. 2019). }\end{array}$ \\
\hline 1.2 & Tenure & $\begin{array}{l}\text { Tenure affects households' investment decisions. The tenure mix of a } \\
\text { neighbourhood affects the overall housing stock }\end{array}$ & Owner vs. occupiers; owner on/off-site; length of stay; fear of eviction & \multirow{4}{*}{$\begin{array}{l}\text { Living Conditions Diamond Gulyani \& } \\
\text { Bassett (2010) }\end{array}$} \\
\hline 1.3 & Housing Unit & $\begin{array}{l}\text { The private domain, linked to residents' development priorities and } \\
\text { technical options }\end{array}$ & Construction of walls, floors, roof; overall building quality & \\
\hline 1.4 & $\begin{array}{l}\text { Infrastructure and } \\
\text { Services }\end{array}$ & Linked to residents' development priorities and technical options & $\begin{array}{l}\text { Is the plot serviced by water, electricity, solid waste collection, street } \\
\text { lighting, paved roads, roads (vehicle access)? }\end{array}$ & \\
\hline 1.5 & Neighbourhood & $\begin{array}{l}\text { To understand the different neighbourhood typologies to be able to } \\
\text { provide appropriate solutions to different neighbourhoods. }\end{array}$ & $\begin{array}{l}\text { Quality of neighbourhood (cleanliness, location, transport, safety/crime, } \\
\text { cost of land) }\end{array}$ & \\
\hline 2 & \multicolumn{4}{|c|}{ THE SERVICE DELIVERY ENVIRONMENT } \\
\hline 2.1 & Containment & \multirow{5}{*}{$\begin{array}{l}\text { To understand the different sanitation options, pathways, and prevalence } \\
\text { of each in the context, including both networked and non-networked } \\
\text { sanitation options. Type of service, equipment, diversification of customer } \\
\text { base; practices; technologies }\end{array}$} & Outcome: Access and use of a hygienic toilet & \multirow{5}{*}{$\begin{array}{l}\text { SFD Protocol (SFD Manual 2018) } \\
\text { Outcome-based sanitation value chain } \\
\text { (Figure 3) }\end{array}$} \\
\hline 2.2 & Emptying & & Outcome: Fecal sludge is safely contained on-site & \\
\hline 2.3 & Transport & & Outcome: Fecal sludge is hygienically removed from containment site & \\
\hline 2.4 & Treatment & & Outcome: Pathogen removal (reduction or inactivation) & \\
\hline 2.5 & Re-use / disposal & & Outcome: Cost and resource recovery & \\
\hline 3 & \multicolumn{4}{|c|}{ THE ENABLING ENVIRONMENT } \\
\hline 3.1 & Policy and Strategy & $\begin{array}{l}\text { To understand if the policy exists and is appropriate to the context and } \\
\text { activities; to understand the direction of travel. }\end{array}$ & Policy, regulation; enforcement; pro-poor; Urban Local Government role & \multirow{5}{*}{$\begin{array}{l}\text { WHO Sanitation Guidelines (2018); } \\
\text { WSUP Enabling Environment (2018); The } \\
\text { World Bank's FSM Diagnostic Tools } \\
\text { (Peal, Evans, Blackett et al. 2014; Ross et } \\
\text { al. 2016), including Citywide Service } \\
\text { Delivery Assessment, UNICEF (2016); } \\
\text { CLUES (Lüthi C., Morel, A. Tilley, E. et al. } \\
\text { 2011); CWIS CSDA (Blackett and Hawkins } \\
\text { 2019) }\end{array}$} \\
\hline 3.2 & $\begin{array}{l}\text { Institutional } \\
\text { Arrangements }\end{array}$ & $\begin{array}{l}\text { To understand the players (both informal and formal) and the rules of the } \\
\text { game, coordination between them }\end{array}$ & Roles and responsibilities; coordination & \\
\hline 3.3 & $\begin{array}{l}\text { Sector Planning and } \\
\text { Monitoring }\end{array}$ & To understand what drive changes & Service targets; monitoring; planning & \\
\hline 3.4 & $\begin{array}{l}\text { Budgeting and } \\
\text { Finance }\end{array}$ & To understand financial planning and procurement processes. & Financial planning and procurement practices and power; cost recovery; & \\
\hline 3.5 & Capacity & $\begin{array}{l}\text { To understand who the decision makers are, their capacity and competing } \\
\text { priorities. }\end{array}$ & $\begin{array}{l}\text { Skills; knowledge; training; resourcing (national to lowest administrative } \\
\text { unit) }\end{array}$ & \\
\hline 4 & \multicolumn{4}{|c|}{ THE INTERFACES AND RELATIONSHIPS } \\
\hline 4.1 & $\begin{array}{l}\text { Key interfaces and } \\
\text { /or relationships }\end{array}$ & $\begin{array}{l}\text { To understand the nature of the relationships, both within, and between } \\
\text { the groups. To highlight gaps and opportunities (i.e., key and missing } \\
\text { relationships and/or interfaces). }\end{array}$ & $\begin{array}{l}\text { What is the relationship/interface/mechanisms of contact, etc between } \\
\text { households (i.e. social cohesion); service providers (e.g., association); and } \\
\text { local-national government actors (e.g., task force)? }\end{array}$ & Relationship mapping; network analysis \\
\hline
\end{tabular}

FIGURE 2 | Proposed Sanitation Cityscape indicators (WHO, 2018).

useful foundation for indicators to describe the way sanitation services are delivered. In Figure 1, the basic services listed are solid waste, drainage, water supply, and sanitation, but these could expand to include other services such as health, education, mobile connectivity, transport, and more, depending on the desired lens of analysis. For sanitation, the Service Delivery Environment assessment should include both formal and informal sanitation providers from across the range of sanitation technologies and service models, including networked and non-networked sanitation systems. We therefore suggest the component stages of the sanitation service chain: capture, containment, transport, treatment, and reuse/disposal are described using five outcome-based indicators respectively to describe the sanitation service environment (see Figure 2).

\section{The Enabling Environment}

The Enabling Environment describes the wider structural and institutional context that frame the service delivery. In the Sanitation Cityscape framework, the Enabling Environment is designated by the outer ring. This placing acts as a reminder that, while the focus of enquiry may be on sanitation, there are inherent dependencies on other basic services; the Enabling Environment for sanitation may involve wider urban governance beyond sanitation. Current Enabling Environment analysis for sanitation typically consists of policy and strategy, institutional arrangements, sector planning and monitoring, and budgeting and finance and capacity. Tools such as the Citywide Service Delivery Assessment (Ross et al., 2016) initially focused only on FSM but have recently been adapted to include both networked and nonnetworked sanitation (Blackett and Hawkins, 2019). The existing tools and approaches to assess the enabling environment for sanitation are comprehensive; therefore, we suggest using the common indicators to describe the Enabling Environment such as policy and strategy, institutional arrangements, sector planning and monitoring, and budgeting and finance and capacity (see Figure 2). Being intentional in setting the boundaries of the Enabling Environment analysis (i.e., as a single or multisector lens and across the appropriate range of service provision) is fundamentally important.

\section{The Interfaces and the Nature of Relationships}

The final concept of the Sanitation Cityscape is understanding some of the relationships within and between these three conceptual environments to highlight interfaces, gaps, and intervention opportunities. The components of this analysis is likely to vary depending on the objective of inquiry, but the aim is to understand the people, the nature of organization, leadership, and cohesion in the Living Environment, and the dynamics between the service providers and decision makers and to identify if there are existing communication channels and interfaces between different groups that can be reinforced (see Figure 2). At this initial stage, just one indicator was used to describe the interfaces and relationships, but there is scope to deploy tools such as network analysis or political economy analysis for deeper insights into these interactions.

\section{Validating the Sanitation Cityscape Framework for Urban Sanitation}

The final stage of Jabareen (2009) process of building a conceptual framework is validation. This is an iterative process to test if the 
framework and its concepts make sense not only to the researcher but also to other scholars and practitioners.

To this aim, the Sanitation Cityscape framework has been presented at a number of sanitation focused workshops and events $^{5}$. Feedback from both practitioners and academics to date has been positive, including comments such as "a useful conceptual framework," "provides clarity," "a useful way of organizing thoughts," "a helpful dashboard" and "a systems approach." This has led to the practical application of the framework to design and assess the baseline situation of the sanitation systems in a town in Ethiopia, Debre Birhan as part of a USAID Sustainable WASH Systems project ${ }^{6}$. The experience of adapting the CSDA tool through the Sanitation Cityscape lens was shared with the CWIS CSDA tool revision team, and the framework has supported the design of several rapid diagnostic assessments of sanitation. The section below offers some conceptual and practical insights from this validation thus far.

\section{Conceptual Insights}

- Alignment to the Citywide Inclusive Sanitation (CWIS) Principals. The Sanitation Cityscape Framework aligns to the Citywide Inclusive Sanitation (CWIS) principals ${ }^{7}$ in that (i) it provides a framework that works for all urban settlements, ranging from informal to formal and specifically integrating tenure as a critical factor of urban development and investment at both the household and public funding level; (ii) it purposefully embraces the complexity of urban environments; (iii) it focuses on outcomes and allowing for a diversity of solutions; and (iv) it embeds sanitation within a wider urban governance framework, where the unit of change becomes the city itself.

- An Outcome-Based Sanitation Service Chain. The sanitation service chain is typically measured using descriptors of

\footnotetext{
5Including the 21st Sanitation Community of Practice (SanCoP) Meeting:
"Addressing the Complexities of Citywide Environmental Sanitation," Leeds, 2018;

${ }^{5}$ Including the 21st Sanitation Community of Practice (SanCoP) Meeting:
"Addressing the Complexities of Citywide Environmental Sanitation," Leeds, 2018; 40th WEDC International Conference, Loughborough, UK, 2017; The IRC All Systems Go! Symposium, The Hague, 2019.

${ }^{6}$ In addition to appropriate socioeconomic indicators such as age, gender, income, etc., as relevant.

${ }^{7}$ Citywide sanitation has been endorsed as the future paradigm for urban sanitation, and several organizations are applying the founding principles of Citywide Inclusive Sanitation (CWIS) in their work.
}

technologies or activities; we suggest that a useful adaption to this is to attribute outcome-based indicators for each stage of the sanitation service chain as an objective comparison across technologies and systems (see Figure 3).

- Data Collection and Sources. Collecting primary data is expensive and time consuming. Several of the existing tools described in this paper rely on extensive primary data collection. The proposed indicator set for the Sanitation Cityscape comprises of 16 indicators, which focuses the data collection to a manageable number. Nevertheless, we suggest that future developments of the tools and the framework consider possible synergies with existing data sets as well as parallel urban development work to further make the best use of available data. This is specifically relevant around indicators that may be shared across urban development initiatives (such as tenure the enabling environment or in area-based approaches) as well as more localized data collection mechanisms including community-led informal settlement profiling initiatives ${ }^{8}$ and other national and subnational monitoring systems.

\section{Insights From the Field}

In 2018, the Sanitation Cityscape was used to design a citywide baseline assessment of the sanitation systems of a small town in Ethiopia, Debre Birhan. The 16 indicators captured data across the three main conceptual environments and the relationships between them using, where possible, standard and validated variables or tools, or adaptations thereof.

The baseline included a representative stratified random household survey $(N=308)$ across the nine administrative units of the town. It deployed the Living Conditions Diamond tool (Gulyani and Basset, 2010) to map the living environment, plus collecting data on residents' development priorities to reflect demand. It collected primary data from key informant interviews and secondary data sources using the SFD protocol to generate an initial fecal flow diagram (Shit Flow Diagram Initiative, 2018), which was validated with stakeholders. It also deployed an adapted version of the CSDA (Ross et al., 2016) to capture data on both FSM as well as the other sanitation services within the

${ }^{8}$ For example https://knowyourcity.info/
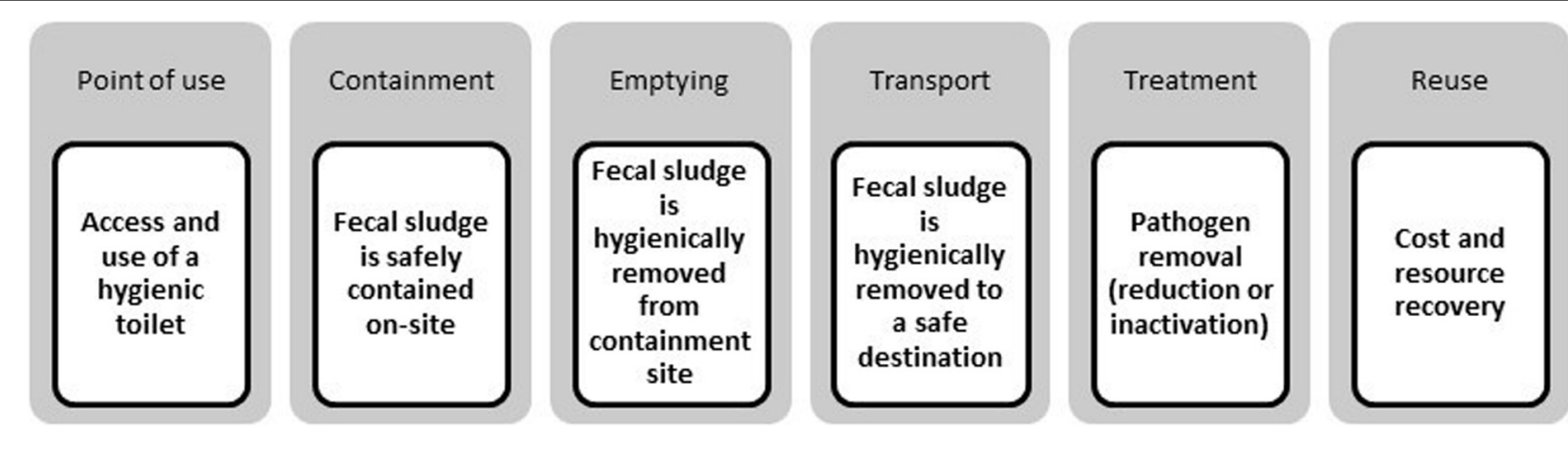

FIGURE 3 | Outcome-based sanitation service chain. 
town (specifically communal and public toilets) (see Figure 2) ${ }^{9}$. To understand the interfaces between the different environments for the Debre Birhan baseline, data were gathered with key informants about the nature and frequency of their relationships with other urban stakeholders. Key insights from applying the framework are summarized below; the baseline results themselves are documented elsewhere (Scott and Henry, 2018).

- Efficiency of Design and Data Collection. The 16 indicators used for the Debre Birhan provided a manageable data set. Data were collected across the three conceptual environments for a town (population of 100,000) by a small research team in $<1$ week. Aggregating the data from a well-defined indicator set that fed into the larger concepts provided a systematic and efficient way of designing the study, collecting, and processing data, and navigating a complex urban sanitation system.

- Neighborhood Typologies. Applying the Living Conditions Diamond analysis across each of the nine administrative units in Debre Birhan generated four settlement typologies with distinct characteristics: one was typical of a central urban neighborhood where residents pay a premium for the location and infrastructure over housing quality, and space is at a premium; another had a tenure mix of more tenants than owner occupiers with a rapid turnover of residents, which is typical of a lower-income urban area; a third typology had a higher ratio of owner occupiers and better housing stock compared to the former two typologies. Identifying area typologies can help identify which type of sanitation service delivery model is likely to be the most appropriate (see Figure 4). In the first two typologies described above, communal

\footnotetext{
${ }^{9}$ At the time of the study, the CSDA was limited to FSM. This has since been updated (Blackett and Hawkins, 2019) with inputs from experience of adapting the CSDA tool through the Sanitation Cityscape lens.
}

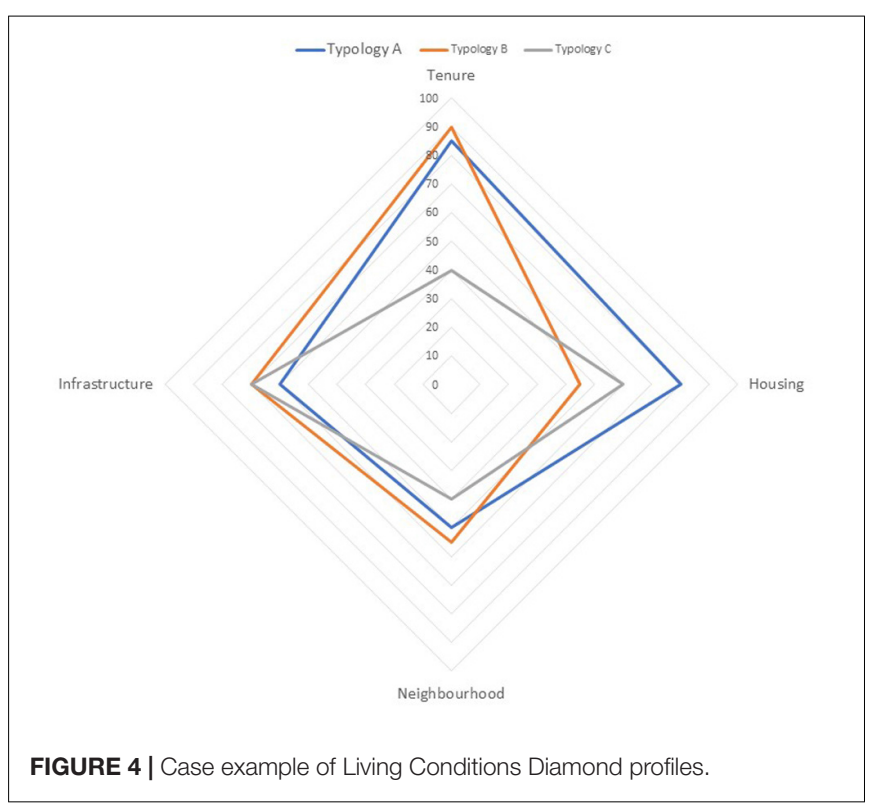

latrines were commonplace. We suggest that an appropriate sanitation response in settlements of that type would likely include service-based models (i.e., shared, public, and container-based toilets) rather than sanitation marketing of individual household toilets. Aligning sanitation responses to settlement typologies is an approach used in the current Guidance on Programming for Rural Sanitation (WaterAid et al., 2019). At the time of the 2018 baseline study, using a similar principle of creating area typologies to align sanitation responses was unique in the urban context. We suggest the Living Conditions Diamond tool, or adaption thereof, might be a useful way to align sanitation responses to urban settlement typologies, or indeed along the urbanrural continuum.

- Boundarying the Enabling Environment. One important finding from the Debre Birhan baseline was that the enabling environments can be very different for different parts of sanitation services. The baseline deployed an adapted version of the CSDA (Ross et al., 2016) to capture data on both FSM as well as the other sanitation services within the town (i.e., communal and public toilets) in parallel (see Figure 2$)^{10}$. What the baseline study showed was a marked difference between the enabling environments of different parts of sanitation services. The enabling environment for FSM was relatively good; thanks to a well-functioning utility, the enabling environment for other sanitation services was much weaker. More recent CWIS revisions of the CSDA tool (Blackett and Hawkins, 2019) have gone some way to address including both networked and non-networked sanitation. However, the fundamental point here is that care needs to be taken to set boundaries purposefully and intentionally for the enabling environment analyses to adequately cover the intended services and governance arrangements.

Key Urban Interfaces. Collecting data around the interfaces between the conceptual environments in Debre Birhan unveiled some "unusual suspects" of sanitation service planning in the town (i.e., those who would not normally emerge in an SFD study or WASH-only enabling environment analysis). Respondents were asked about the nature and frequency of interactions with actors in the other environments. Interview responses highlighted the pivotal role of officers at the lowest administrative units, specifically the highest ranking official as well as the health extension and enforcement officers on the front line. This workforce is often overlooked and underresourced but were identified as critical in terms of sustaining urban sanitation provision for both achieving access and maintaining environmental health. Edirs, or self-organized community savings groups ${ }^{11}$, also emerged as key interfaces at

\footnotetext{
${ }^{10}$ At the time of the study, the CSDA was limited to FSM. This has since been updated (Blackett and Hawkins, 2019) with inputs from experience of adapting the CSDA tool through the Sanitation Cityscape lens.

${ }^{11}$ Typically used to cover funeral costs and support deceased families, the Debre Birhan baseline found Edirs were being used to support development activities such as building houses for the poorest members of the community.
} 
the community level. This is noteworthy as the ability of an urban community to self-organize into savings groups has been identified a proxy for social cohesion and a predeterminant for public finance for local development initiatives (Bhatkal and Lucci, 2015). Finally, large-scale private sector such as hotels and breweries were identified through key informant interviews about the relationships between urban actors as largest polluters but also the largest employers in the town, presenting challenges in terms of power dynamics.

\section{CONCLUSION}

The last decade has seen great advances in the understanding of the Sanitation Service Delivery Environment, but the "business as usual" approach for the sanitation sector will not achieve SDG 6.2 and 6.3. There needs to be a substantial effort to understand the wider human-technology-environment systems at play and embed sanitation into urban governance. Citywide Inclusive Sanitation has been endorsed as the future paradigm for urban sanitation, and several organizations are applying the founding principles in their work. There is, as yet, however, little consistency in how urban sanitation systems are described and measured.

For the researcher and practitioner alike, the Sanitation Cityscape narrows the complexity of urban sanitation into three main concepts: the Living Environment, the Service Delivery Environment, and the Enabling Environment. We suggest that it is the aggregation of the concepts that adds value, providing a coherent "frame" to locate the components of the urban sanitation puzzle predictably and systematically. The framework and indicator set lends itself to rapid diagnostic analysis and more appropriate targeting of appropriate sanitation interventions. By breaking the urban sanitation system down into conceptual environments, there is scope, within each conceptual environment, for a deeper granularity of analysis: the living and service delivery assessments indicate what are priority areas for urban sanitation. It locates the importance of sanitation in relative terms to other urban development issues. The enabling environment analysis lends insights as to why the situation is the way it is. Finally, examining the interfaces and relationships

\section{REFERENCES}

Bhatkal, T., and Lucci, P. (2015). Community-Driven Development in the Slums: Thailand's Experience. ODI Development Progress Case Study Summary. Available online at: https://www.odi.org/sites/odi.org. uk/files/odi-assets/publications-opinion-files/9669.pdf (accessed June 22, 2020).

Blackett, I., and Hawkins, P. (2019). City Service Delivery Assessment for Citywide Inclusive Sanitation. Available online at: https://www.susana.org/ en/knowledge-hub/resources-and-publications/library/details/3700 (accessed June 22, 2020).

Cairncross, S. (1992). Sanitation and Water Supply: Practical Lessons from the Decade. Washington, DC: The World Bank.

Chambers, R. (1983). Rural Development: Putting the Last First. Oxford: John Wiley and Sons.

Cotton, A., and Franceys, R. (1987). Services for Urban Low-Income Housing, National Housing Development Authority of Sri Lanka. Loughborough: Water, Engineering and Development Centre. between the different conceptual environments helps to highlight how things can change, identifying potential intervention points or system levers. Considered together, the three conceptual environments provide useful insights into what, why, and how to address sanitation as an urban governance issue.

Building a conceptual framework is an iterative process, and we welcome new insights or developments to further validate the Sanitation Cityscape framework. We have proposed a set of 16 key indicators in this paper, although these are not intended to be prescriptive; rather, we suggest the approach of locating them within the conceptual environments is a useful one. The validation efforts to date suggest that the Sanitation Cityscape offers a useful framing in moving toward Citywide Inclusive Sanitation and embedding sanitation within urban governance frameworks. We hope the framework might provide a foundation for greater consistency and a common vocabulary around the fundamental concepts and indicators relevant to urban sanitation. This, in turn, we hope might lead to more purposeful inquiry and cumulative knowledge base, both within and beyond the sanitation sector, that serves multiple sets of interests.

\section{DATA AVAILABILITY STATEMENT}

The datasets generated for this study are available on request to the corresponding author.

\section{AUTHOR CONTRIBUTIONS}

PS developed the conceptual framework in consultation with AC. Both authors wrote the manuscript.

\section{ACKNOWLEDGMENTS}

We would like to extend thanks to the Tetra Tech and the Sustainable WASH Systems Learning Partnership for sharing the data collected from the Sustainable WASH Systems Learning Partnership 2019 baseline study from Debre Birhan, Ethiopia. For more information, visit www.globalwaters.org/SWS.

Cotton, A., and Franceys, R. (1991). “Services for Shelter," in Liverpool Planning Manual 3, ed. G. Dix (Liverpool: Liverpool University Press in association with Fairstead Press).

Devine, J. (2009). Introducing SaniFOAM: A Framework to Analyze Sanitation Behaviors to Design Effective Sanitation Programs. Water and Sanitation Program: Working Paper. Washington DC: The World Bank.

Dreibelbis, R., Winch, P. J., Leontsini, E., Hulland, K. R., Ram, P. K., Unicomb, L., et al. (2013). The integrated behavioural model for water, sanitation, and hygiene: a systematic review of behavioural models and a framework for designing and evaluating behaviour change interventions in infrastructure-restricted settings. BMC Public Health 13:1015. doi: 10.1186/ 1471-2458-13-1015

Gulyani, S., and Basset, E. (2010). The living conditions diamond: an analytical and theoretical framework for understanding slums. Environ. Plann.A 42, 2201-2219. doi: 10.1068/ a42520

IWA (2006). Sanitation 21. Simple Approaches to Complex Sanitation. A Draft Framework for Analysis. London: International Water Association. 
Jabareen, Y. (2009). Building a conceptual framework: philosophy, definitions, and procedure. Int. J. Q. Methods 8, 49-62. doi: 10.1177/160940690900 800406

Jenkins, M. W., and Scott, B. (2007). Behavioral indicators of household decisionmaking and demand for sanitation and potential gains from social marketing in Ghana. Soc. Sci. Med. 64, 2427-2442. doi: 10.1016/j.socscimed.2007.03.010

Kalbermatten, J., Middleton, R., and Schertenleib, R. (1999). Household Centred Environmental Sanitation. Dübendorf: Swiss Federal Institute for Environmental Science and Technology.

Kar, K. (1997). Participatory Impact Assessment: Calcutta Slum Improvement Project, New Delhi. Available online at: https://www. environmentandurbanization.org/participatory-impact-assessment-calcuttaslum-improvement-project (accessed June 22, 2020).

Lüthi, C., Morel, A., Tilley, E., and Ulrich, L. (2011). Community-Led Urban Environmental Sanitation Planning - Complete guidelines for Decision-Makers With 30 Tools. Available online at: https://www.eawag.ch/fileadmin/Domain1/ Abteilungen/sandec/schwerpunkte/sesp/CLUES/CLUES_Guidelines.pdf (accessed June 22, 2020).

Miles, M., and Huberman, M. (1994). "Qualitative Data Analysis: An Expanded Sourcebook, 2nd Edn. Beverley Hills, CA: Sage.

Parkinson, J., Lüthi, C., and Walther, D. (2014). Sanitation 21 - A Planning Framework for Improving City-Wide Sanitation Services. Caxton St: IWA.

Peal, A., Evans, B., Blackett, I., Hawkins, P., and Heymans, C. (2014). Fecal sludge management: analytical tools for assessing FSM in cities. J. Water Sanitat. Hygiene Dev. 4, 371-383. doi: 10.2166/washdev.2014.139

Ross, I., Scott, R., Blackett, I. C., and Hawkins, P. M. (2016). Fecal Sludge Management: Diagnostics for Service Delivery in Urban Areas Summary Report. Available online at: http://documents.worldbank.org/ curated/en/909691468338135561/Fecal-sludge-management-diagnosticsfor-service-delivery-in-urban-areas-summary-report (accessed June 22, 2020).

Scott, P. (2019). The Sanitation Cityscape Conceptual Framework - Understanding Urban Sanitation Systems, Proceedings of the All Systems go! Symposium, The Hague, 2019. Available online at: https://www.ircwash.org/sites/default/files/ 084-201906scott.pdf (accessed June 22, 2020).

Scott, P., Cotton, A., and Sohail, M. (2015). Using tenure to build a "sanitation cityscape": narrowing decisions for targeted sanitation interventions. Environ. Urbaniz. 27, 389-406. doi: 10.1177/09562478155 69415

Scott, P., and Henry, L. (2018). Sanitation in Small Towns - Debre Birhan, Ethiopia: Baseline Assessment Report. Sustainable WASH Systems Learning Partnership Research Report. Available online at: https://files.globalwaters.org/waterlinks-files/SWS\%20Debre\%20Birhan\%20Baseline\%20Assessment\%202018.pdf (accessed June 22, 2020).

Scott, P., Scott, R., and Cotton, A. (2017). "Urban sanitation: where to next?," in 40th WEDC International Conference, Loughboroug.
Shit Flow Diagram Initiative (2018). SFD Manual Vol. 1 and 2. Available online at: https://sfd.susana.org/knowledge/the-sfd-manual (accessed June 22, 2020).

Tidwell, J., Terris-Prestholt, F., Quaife, M., and Aunger, R. (2019). Understanding demand for higher quality sanitation in peri-urban Lusaka. Zambia through stated and revealed preference analysis. Soc. Sci. Med. 232, 139-147. doi: 10. 1016/j.socscimed.2019.04.046

Tilley, E., Ulrich, L., Lüthi, C., Reymond, P., and Zurbrügg, C. (2008). Compendium of Sanitation Systems and Technologies. Dübendorf: Water Supply and Sanitation Collaborative Council (WSSCC).

UNICEF (2016). Strengthening Enabling Environment For Water, Sanitation And Hygiene (WASH) Guidance Note. Available online at: https: //washenablingenvironment.wordpress.com/guidance/ (accessed June 22, 2020).

WaterAid, UNICEF, and Plan International (2019). Guidance on Programming for Rural Sanitation. Available online at: https://washmatters.wateraid.org/sites/ g/files/jkxoof256/files/guidance-on-programming-for-rural-sanitation.pdf (accessed June 22, 2020).

WHO (2015). Sanitation Safety Planning Manual for Safe use and Disposal of Wastewater, Greywater and Excreta. Geneva: World Health Organization.

WHO (2018). Guidelines on Sanitation and Health. Geneva: World Health Organization.

WHO/JMP (2016). Safely Managed Sanitation Services. Available online at: https://www.who.int/water_sanitation_health/monitoring/coverage/ explanatorynote-sdg-621-safelymanagedsanitationsServices161027.pdf (accessed June 22, 2020).

Wright, A. (1997). Toward a Strategic Sanitation Approach: Improving the Sustainability of Urban Sanitation in Developing Countries. Washington DC: The World Bank.

WSUP (2018). Enabling Environments for Inclusive Citywide Sanitation: a Conceptual Framework. Water and Sanitation for the Urban Poor. Available online at: https://www.wsup.com/blog/enabling-environments-for-inclusivecitywide-sanitation-a-conceptual-framework (accessed June 22, 2020).

Conflict of Interest: The authors declare that the Sanitation Cityscape Framework was developed in the absence of any commercial or financial relationships that could be construed as a potential conflict of interest. The data of the 2018 Debre Birhan baseline that deployed the Sanitation Cityscape Framework was under the auspices of the Sustainable WASH Systems Learning Partnership and funded by USAID.

Copyright (c) 2020 Scott and Cotton. This is an open-access article distributed under the terms of the Creative Commons Attribution License (CC BY). The use, distribution or reproduction in other forums is permitted, provided the original author(s) and the copyright owner(s) are credited and that the original publication in this journal is cited, in accordance with accepted academic practice. No use, distribution or reproduction is permitted which does not comply with these terms. 